\title{
Responsabilidad social universitaria y la gestión institucional
}

\author{
Lady Diana Arévalo Alva \\ Ladydianaarevaloalca85@gmail.com \\ Carlos Miguel Aguilar Saldaña \\ Agua0122@hotmail.com
}

Rosa Elena Cueto Orbe rosacueto.69@gmail.com

Luis Alberto Ríos López

Rioslopezluisalberto09@gmail.com

Sandra Ruiz Corea

sruiz_correa@hotmail.com

Blanca Yalta Flores

Blancayaltaflores62@gmail.com

Escuela de post grado Universidad César Vallejo.

\section{RESUMEN}

La investigación se centra esencialmente en establecer la relación que existe entre la responsabilidad social universitaria y la gestión institucional de la Universidad Nacional de San Martin sede Tarapoto. Se justifica ya que la proyección social de las universidades no es sólo acción externa, sino que tiene incidencia académica a través de la introducción de cambios en el currículo y en la pedagogía. El objetivo es determinar la relación que existe entre la responsabilidad social universitaria y la gestión institucional de la Universidad Nacional de San Martin. La investigación presenta un diseño descriptivo correlacional, ya que se procedió al describir las variables en función a los resultados, luego se estableció la relación existente entre las mismas. Como técnicas de recolección de datos se ha establecido la encuesta. Se llegó a concluir, que existe una relación significativa entre las variables, ya que se evidencia una relación positiva muy fuerte, pues el coeficiente de correlación es de 0.807 ; este coeficiente, al ser elevado al cuadrado, evidencia que la variable I incide o afecta en un $65.12 \%$ sobre la variable II.

Palabras clave: gestión; responsabilidad; universitaria; social. 


\title{
University social responsibility and institutional management
}

\begin{abstract}
The research essentially focuses on establishing the relationship that exists between university social responsibility and institutional management at the National University of San Martin, Tarapoto headquarters. It is justified since the social projection of the universities is not only external action, but also has an academic impact through the introduction of changes in the curriculum and in pedagogy. The objective is to determine the relationship that exists between university social responsibility and institutional management of the National University of San Martin. The research presents a correlational descriptive design, since the variables were described based on the results, then the existing relationship between them was established. The survey has been established as data collection techniques. It was concluded that there is a significant relationship between the variables, since there is a very strong positive relationship, since the correlation coefficient is 0.807 ; this coefficient, when squared, shows that variable I affects or affects variable II by $65.12 \%$.
\end{abstract}

Keywords: management; responsibility; university; social.

Artículo recibido: 10. Junio. 2021 Aceptado para publicación: 16. Julio. 2021 Correspondencia: Ladydianaarevaloalca85@gmail.com

Conflictos de Interés: Ninguna que declarar 


\section{INTRODUCCIÓN}

Todas las universidades en el mundo se enfrentan a un conjunto de procesos de cambios generados por muchas veces por factores políticos. La prioridad de toda universidad es demostrar que su razón de ser está orientado a resolver problemas y necesidades dentro de la sociedad. Para ello todas y cada una de las instituciones y organizaciones buscan mantener una buena gestión sobre las actividades que realizan, para ello recurren a una serie de procedimientos en los cuales evalúan todos y cada uno de los procesos realizados, con el único fin de detectar un problema que deba ser solucionado, ya que la universidad buscar ser competitivo. La universidad peruana para insertarse en un escenario competitivo es necesario plantear estrategias que conduzcan a superar retos como: mejorar la capacitación del personal, distribución equitativa de los recursos, calidad de la enseñanza, investigación y mejores servicios, pertinencia de los planes de estudio, mayores posibilidades de empleos para los egresados; por lo que la Universidad Nacional de San Martín a través de la Facultad de Ciencias Económicas no es ajena a esta realidad. Con la promulgación de la Ley N 28740 (Ley del SINEACE), se inició el camino a la acreditación de la calidad de las Instituciones Educativas y de sus programas, siendo las Universidad y sus carreras profesionales y programas de Pos Grado competencia del Consejo Nacional de Evaluación, Acreditación, Certificación de la Calidad de la Educación Universitaria - SINEACE.

La Universidad Nacional de San Martin sede Tarapoto, presenta problemas frecuentes en el aspecto organizacional y sobre todo educativo, al mismo tiempo presenta problemas en el aspecto cognitivo como es la difusión y transferencia de conocimientos y promoción de investigaciones, y no hay que dejar de lado los problemas sociales, como es la participación activa de todos los involucrados y para poder mejorar se requiere como pilar fundamental la Responsabilidad para con la Sociedad y para con el entorno natural en la que se encuentra inserta, en otras palabras hablamos de Responsabilidad Social Universitaria. A pesar de problemas existentes provenientes del factor externo por los malos manejos de las políticas educativas de los gobiernos nacionales, la universidad de San Martin viene trabajando pensando en los alumnos y la sociedad, donde el compromiso que tiene la universidad con la responsabilidad social es grande. Es muy importante también mencionar falencias en la gestión institucional por la falta de liderazgo directivo, falta de una buena planificación estratégica y sobre todo 
el desempeño docente, y ello hace que el clima institucional se vea afectado y todos los actores involucrados no estén dando un buen desempeño.

\section{Responsabilidad social universitaria}

Domínguez y Ramos (2012), sostienen que la responsabilidad social universitaria, consiste en la entrega de un servicio social, como consecuencia del proceso de enseñanza y aprendizaje, respondiendo al principio universal de hacer el bien, y de compartir con los demás lo que se ha realizado. Esta responsabilidad es el producto de una gestión basada en la ética y la efectividad de las actividades, con la finalidad de superar la gestión organizacional.

Aldeanueva et al. (2015), manifiestan que la responsabilidad social universitaria (RSU) implica el potenciar la contribución de cada una de las universidades al desarrollo social y económico de la población en las que se encuentran. La RSU se encuentra fundamentado en tres ejes o ámbitos fundamentales, estos son el eje económico, eje ocia, y ejes medioambientales.

Siglo 21 (2013), constituye un desafío a las universidades, ya que implica que las universidades se comprometan no solo con la formación de profesionales de bien, sino también d la formación de personas empáticas con los problemas sociales, comprometidas con el cambio y el desarrollo de su territorio y país, así como de la inclusión social, etc.

Castro (2014) refiere que la responsabilidad universitaria es el despliegue y aplicación de una serie de normas cuyo fin principal es la vinculación entre la universidad y la sociedad, se encuentra encaminada al crecimiento de ambas muestras en función a la investigación, formación profesional y la difusión. La finalidad de un modelo de RSU, son los siguientes: Dar sustento teórico a la RSU. Implementar políticas y estrategias que respondan a la realidad nacional. Orientar las prácticas de responsabilidad social en la universidad, mediante una propuesta acorde con la identidad de la UNMSM. Incluir la RSU como eje transversal en el diseño curricular de las carreras.

\section{Evaluación de la responsabilidad social universitaria}

Impactos universitarios

Vallaeys, De la Cruz y Sasia (2014). Refieren que las universidades cuyo objetivo es promover la enseñanza a la sociedad, por ende, para el análisis de la responsabilidad 
social de la universidad es el entorno del mismo, las cuales presentan cuatro categorías, (educativa, organizacional, social y cognitiva).

Impactos organizacionales

Es el impacto que genera la universidad en los colaboradores administrativos con que cuenta, las cuelas están conformados, por el personal administrativo, así como docentes a la ves estudiantes, además generan impactos ambientales en los culés podemos encontrar (forestación, trasporte, desechos entre otros.)

Difusión del concepto de RSU: el cual va dirigido la comunidad universitaria, por ello comprende el desarrollo y difusión de la calidad de RSU que presenta la universidad en el entorno interno, con la finalidad de aumentar el porcentaje de personas que conozcan de una manera óptima las políticas manejadas por la universidad, con el fin de promover una mejor enseñanza.

Derechos humanos: comprende la equidad de género y no discriminación, estas las normas de éticas internas manejadas por la institución, las mismas que se encuentran monitoreados por un comité, quien se asegura del cumplimiento de los códigos de ética manejas por la institución. Por ello toda institución debe regirse por códigos de ética para no generar discriminación, más porcentajes de mujeres en cargos administrativos.

Desarrollo personal y profesional. Generación de un buen clima laboral es lo primordial para toda institución ya que de ello depende la calidad del entorno interno, por ello en toda institución es de importancia la existencia de medidas correctivas, las culés son aplicables una vez detectada las deficiencias, toda institución debe promover la mejora del clima laboral atreves de reuniones, participaciones entre otros.

Gestión educativa. Existencia de educación y gestión ambiental, a través de la selección óptima de proveedores las culés deben estar de acuerdo a los criterios de responsabilidad tanto social, así como ambiental.

Buen gobierno universitaria. Una gestión adecuada permite los logros de la institución a nivel social, ambiental, así como educativo. El mismo que debe ser acompañado mediante la trasparencia economía.

Comunicación y marketing. Campañas que promueven los temas sociales de la institución, las cuales deben realizar de forma responsable.

\section{Impactos educativos}

La universidad es el encargado de instituir valores así como la formación profesional, por medio del cual todo profesional se forma profesionalmente, el mismo que lo permite 
a ver el mundo y aprender a convivir en él, la universidad es el encargado de formar profesionales por ende es el responsable de poner al mercado la calidad de profesionales de cada una de las disciplinas acorde a las necesidades la sociedad, es por ello de importancia la organización que lleva la universidad por el medio del cual le permita garantizar la calidad de la enseñanza.

Responsabilidad social: Comprende la Inclusión de temáticas ciudadanas y de responsabilidad social en el currículo, estos temas manejados por la institución deben estar enmarcados al logro de los objetivos fijados por el milenio

Articulación: La articulación debe realizarse entre profesionalización y voluntariado solidario, desarrollo de políticas estudiantiles juntamente con el personal administrativo y docentes, así como promover proyectos de emprendimiento.

Aprendizaje profesional: La cual debe ser basado en proyectos sociales, por ello es fundamental incentivar proyectos que estén vinculados a proyectos sociales, por ende la existencia de la cantidad de cursos orientados a la proyección social, el mismo que genere el desarrollo de los proyectos sociales, así mismo se debe desarrollar la capacitación a docentes orientados al mismo.

Integración de actores sociales externos: Las cuales deben integrarse en el diseño de las mallas curriculares, ya que estas son vínculos de la institución con el sector exterior a través de sus facultades, las mismas que le permiten desarrollar proyectos para la mejora de la sociedad.

\section{Impactos cognitivos}

El objetivo que persigue toda universidad es la producción del conocimiento, siguiendo la línea de la verdad en lo que respecta a la legitimidad, utilidad, enseñanza, etc., la universidad incentiva los saberes por cada especialidad llevando de la mano la articulación entre la sociedad y la tecnología, con la finalidad de mejorar el conocimiento, es por ello toda universidad se preocupa por la calidad del conocimiento que produce para la sociedad.

Promoción de la inter y transdiciplinariedad: Desarrollo de talleres de capacitación para docentes de distintas áreas de investigación.

Integración de actores sociales externos en el diseño de líneas de investigación: Desarrollo de convenios con carácter de cooperación entre las instituciones de 
investigación universitarias, así como con los actores externos con la finalidad de mejora en la investigación social.

Difusión y transferencia de conocimientos: Las cuales deben ser socialmente útiles hacia públicos desfavorecidos, producción de documentos publicados por año, por ende, la existencia de políticas manejadas por la institución en promover el conocimiento y tecnología a los sectores de la sociedad menos favorecidas.

Promoción de investigaciones: Estas promociones deben estar aplicadas a temas de desarrollo, como el desarrollo de proyectos con la colaboración de la administración pública.

\section{Impactos sociales}

La universidad es el actor principal de llevar o no al desarrollo a la sociedad, promover profesionales capaces de generar el desarrollo, así mismo preparar al alumno para que tenga un mayor vínculo con lo exterior, por el medio del cual busca la accesibilidad de la enseñanza para todos. Es por ello toda universidad se pregunta la manera de fomentar y/o acompañar el desarrollo toda una sociedad, así como identificar los problemas y ayudar a resolverlo el mismo.

Proyección social. Comprende la integración de la formación académica con la proyección social, así como desarrollo de políticas que estén articulados con la extensión universitaria, así como con la formación academia y científica. En donde los proyectos estén encaminados a lo social

Lucha contra el asistencialismos y paternalismo: Desarrollo de estrategias para la evaluación de los proyectos sobre el impacto de cada uno de ellos. Así como el desarrollo de estrategias para el seguimiento de los proyectos de investigación de los estudiantes.

Promoción de redes sociales: Estas deben estar direccionados a lograr el desarrollo, así como el desarrollo de estrategias las culés permitan convenios con las instituciones del exterior en temas de desarrollo social, así como ambiental.

Participación activa: La participación debe ser activa tanto en la agenda local y nacional de actividades con la finalidad de promover el desarrollo en los sectores ambientales, así como en sectores sociales a través de la utilización de medios masivos, así como el desarrollo de convenios con instituciones públicas con la finalidad de promover el desarrollo social y ambiental. 


\section{Gestión institucional}

Instituto Dominicano de Evaluación e Investigación de la Calidad Educativa (IDEICE, 2011), concibe a la gestión institucional como el conjunto de procesos y acciones que son desarrolladas dentro del campo administrativo, que sirve como base y apoyo a la gestión educativa de una institución. Entre los principales ejes se encuentran:

- La formación y desarrollo del personal (maestros, técnicos y líderes).

- Búsqueda y aprovisionamiento de insumos materiales y de recursos de aprendizaje.

- Relación dinámica entre la parte pedagógica y administrativa.

- Gestión de los órganos de participación y de representación escolar, etc.

Ruiz (2015) sostiene que la gestión universitaria implica el impulsar la dirección de la institución educativa, hacia metas establecidas a partir de una planificación educativa, para lo cual es necesario el contar con conocimientos, habilidades y experiencias respecto al medio en el cual se desarrollan. Una adecuada planificación es importante en la medida de que permite el desarrollo de las acciones de conducción-administración y gestión, ya sean educativas o escolares.

UNESCO-Perú (2011), es el conjunto articulado de las características, políticas y procesos de la organización que ha de llevar a la práctica el planteamiento pedagógico que se propone desarrollar la comunidad educativa.

Evaluación de la gestión institucional. Elera (2010), quien cita a (Huari, 2007) menciona que la gestión institucional son las actividades realizadas de manera interna basándose en el principio de la planificación, y cuyas repercusiones buscan el bienestar de la organización, así como de sus demás elementos. De este autor se rescata las siguientes dimensiones a tener en cuenta respecto a la gestión institucional:

Liderazgo directivo. Constituye la capacidad que tienen las personas a la cabeza de la organización de servir como ejemplos, mediante la realización de ciertas actividades como las que se mencionan a continuación:

Toma de decisiones. Constituye la capacidad de tomar la decisión más adecuada frente a una determinada situación, beneficiando a las partes que intervienen dentro de este proceso.

Estilo de liderazgo. Comprende la forma bajo la cual la persona (directivo), maneja y gestiona a los componentes bajo su responsabilidad, el estilo de liderazgo más aceptado 
es el democrático, sin embargo, ello no quiere decir que se presenten estilos como el autocrático, liberal, sobreprotector, entre otros.

Participación y trabajo en equipo. Constituye la capacidad de trabajar con un grupo de personas, integrándose de manera tal que todos se sientan comprometidos y alcances las metas establecidas.

Planificación estratégica. Para (Elera, 2010) es la identificación de aquellas situaciones indeseables y perjudiciales dentro de la institución, lo que conlleva a una posterior identificación de soluciones, según la gravedad del caso. Los indicadores a tener en cuenta son los siguientes:

Diagnostico. Se evalúa si la persona o responsable realiza un diagnostico acerca de los problemas que se presentan dentro de la institución. Dentro de este diagnóstico se contemplan problemas anteriores, como base para detectar un problema.

Elaboración de proyectos. Constituye la elaboración de las posibles soluciones a los problemas identificados. Se toma en cuenta, los recursos que se emplearan, así como el tiempo estimado en el cual se prevé que se otorgara solución al problema.

Priorización del problema. Si bien es cierto todos los problemas son malos para la organización es prioritario evaluar y priorizar aquellas que presentan mayor relevancia, y que por ende deben de ser solucionados a corto plazo, ello con la finalidad de incrementar el nivel de riesgo dentro de la empresa.

Clima institucional. Es el conjunto de comportamientos que presenta una determinada institución o ambiente, y bajo el cual se moldea una forma de trabajar que de salirse de control puede provocar que el trabajo no sea cumplido de manera efectiva. Bajo lo mencionado por Elera (2010), los indicadores del clima institucional son:

Relaciones interpersonales. Conjunto de conductas, comportamientos y formas de coexistir entre los diferentes miembros del equipo de trabajo de la institución. En este se punto se toma en cuenta las relacione que se generan, el trato, la aplicación de valores como el compañerismo, solidaridad, etc.

Solución de conflictos. Constituye la capacidad de los integrantes para dar solución a los problemas o conflictos que se presentan dentro de la institución o dentro de su competencia. La manera como lo solucionan es importante, ya que, de quedarse alguna rencilla o rencor entre las partes, el clima organizacional se deteriora.

Desempeño docente. Para Elera (2010) refiere que el desempeño docente es la evaluación final que se realiza al grupo humano de docentes encargados de impartir la 
educación dentro de la institución. Para tales efectos, es necesario que se tenga en consideración dos puntos o indicadores importantes, estos son:

Planificación curricular. Constituye la iniciativa del docente para estructurar los temas a tratar en cada una de las unidades que se encuentran distribuidas en un periodo determinado. Ello con la finalidad de entregar un servicio y educación adecuada a los clientes (estudiantes).

Planificación de la clase. Se evidencia cuando el docente planifica y sus sesiones de clase con antelación, la misma que debe de encontrarse de manera congruente con la planificación curricular previamente establecida. Así mismo, se evalúa en la capacidad que tiene el docente para prever los materiales a emplear para el desarrollo de la actividad.

\section{ESTRATEGIAS METODOLÓGICAS O MATERIALES Y MÉTODOS}

Se planteó una investigación correlacional, se aplicó una encuesta y el instrumento de recolección de datos es un formulario tipo cuestionario de 36 preguntas, para responsabilidad social universitaria y 17 preguntas para el tema de Gestión Institucional con una escala Likert. Luego, se procedió al describir las variables en función a los resultados y se estableció la relación existente entre las mismas. La confiabilidad de los instrumentos se llevó por medio del programa Alfa Crombach. Para el análisis se empleará el software estadístico SPSS V.21 y según las escalas de medición de ambas variables. Los estadísticos serán: Tabla de frecuencia para datos agrupados, prueba de Sperman.

\section{RESULTADOS Y DISCUSIÓN}

\section{RESULTADOS}

\section{Responsabilidad social universitaria de la Universidad Nacional de San Martin sede Tarapoto.}

En cuanto a la dimensión impacto organizacional, esta es calificada por el $54.0 \%$ de los colaboradores como adecuada, ya que dentro de la universidad se cumple con los códigos de ética establecidos, tomando las debidas medidas correctivas frente a la presencia de un problema, deficiencia o falta. Se promueve además la participación de mujeres dentro de los puestos organizacionales sin discriminación alguna. Otro de los factores es que se establecen y desarrollan estrategias que facilitan el estado situacional de la cultura y clima organizacional; Se desarrollan campañas para sensibilizar sobre temas ambientales dentro de la universidad. 
La dimensión impacto educativo de la variable responsabilidad social universitaria es catalogada como adecuada en un 54.4\%, ello debido a que la mayor parte de los colaboradores sostiene que en ocasiones posible observar que dentro de las áreas, se dictan cursos relacionados con la responsabilidad social, promoviendo además políticas que buscan el voluntariado en docentes y estudiantes. Así mismo, es notable evidenciar el esfuerzo que se dispone al promover proyectados hacia la responsabilidad social por todo un año.

En cuanto al impacto cognitivo, este es adecuado en un 52.3\%, debido a que una parte de los colaboradores menciona que se fomenta la investigación a través de grupos interdisciplinarios, así como proyectos en los cuales se cuenta con la participación y colaboración de entidades públicas, con el fin de atender a los problemas sociales locales.

La última dimensión de la responsabilidad social universitaria, es el impacto social, el cual es calificado como adecuado en un $52.7 \%$, ello debido fundamentalmente a que se desarrollan estrategias que contribuyan con un adecuado monitoreo de las actividades así como de los proyectos enfocados en la solución o explicación de fenómenos y problemas tanto sociales como ambientales. De esta manera, un factor adicional, es la promoción de estos proyectos en medios masivos, con la finalidad de informar a la población acerca de las actividades que se encuentran realizando los estudiantes de la universidad de forma conjunta con el personal de la institución.

De manera general, la responsabilidad social universitaria ha recibido una calificación de adecuada por la mayoría de los colaboradores encuestados, quienes representan alrededor del $59.1 \%$, este resultado se debe a que se evidencia no solo la preocupación de la institución por los problemas sociales y ambientales dentro del medio, sino que además, se evidencia los deseos de contribuir de alguna manera, mediante la participación activa de todos sus componentes (docentes, personal administrativo, estudiantes).

\section{Gestión institucional de la Universidad Nacional de San Martin sede Tarapoto.}

La gestión institucional, es calificada como adecuada en un $56.5 \%$, ello debido a que, en su mayoría, los colaboradores mencionan que las decisiones que se toman dentro de la institución son adecuadas, ya que benefician a las partes involucradas, por medio de la gestión responsable y la asignación de ciertas responsabilidades encaminadas al 
cumplimiento de las metas. Frente a la presencia de un sin número de proyectos, las autoridades competentes se encargan de priorizar aquellas con mayor relevancia y urgencia. Así mismo, se busca mejorar el clima laboral a través de estrategias organizacionales, y en cuanto a los docentes se refiere, en su mayoría estos elaboran sus clases con anticipación de forma responsable.

Relación de Responsabilidad Social Universitaria-Gestión Institucional de la Universidad Nacional de San Martin sede Tarapoto.

Tabla 1: Prueba de correlación Responsabilidad Social Universitaria-Gestión Institucional

\begin{tabular}{|c|c|c|c|c|}
\hline & & & $\begin{array}{c}\text { Responsabilidad } \\
\text { Social } \\
\text { Universitaria } \\
\end{array}$ & $\begin{array}{c}\text { Gestión } \\
\text { Institucional }\end{array}$ \\
\hline \multirow{6}{*}{$\begin{array}{l}\text { Rho de } \\
\text { Spearman }\end{array}$} & \multirow{3}{*}{$\begin{array}{l}\text { Responsabilidad } \\
\text { Social } \\
\text { Universitaria }\end{array}$} & $\begin{array}{l}\text { Coeficiente de } \\
\text { correlación }\end{array}$ & 1,000 &, $807^{* *}$ \\
\hline & & Sig. (bilateral) & . & ,000 \\
\hline & & $\mathrm{N}$ & 237 & 237 \\
\hline & \multirow{3}{*}{$\begin{array}{l}\text { Gestión } \\
\text { Institucional }\end{array}$} & $\begin{array}{l}\text { Coeficiente de } \\
\text { correlación }\end{array}$ &, $807^{* *}$ & 1,000 \\
\hline & & Sig. (bilateral) & ,000 & . \\
\hline & & $\mathrm{N}$ & 237 & 237 \\
\hline
\end{tabular}

Fuente: Cuestionario aplicado a 237 trabajadores de la Universidad Nacional de San Martín.

Elaboración: Propia

$\mathrm{r} \quad$ : Coeficiente de correlación $=0,807$

$\mathrm{r}^{2} \quad$ : Coeficiente determinante $=0,807^{2}$

$\mathrm{r}^{2} \quad$ : Coeficiente determinante $=65.12 \%$

Se ha determinado que existe una relación significativa entre las variables, pues el valor sig. Obtenido es de 0.000 , así mismo, se evidencia una relación positiva muy fuerte, pues el coeficiente de correlación es de 0.807; este coeficiente, al ser elevado al cuadrado, evidencia que la variable I incide o afecta en un $65.12 \%$ sobre la variable II.

\section{DISCUSIÓN}

La responsabilidad social universitaria, es el esfuerzo que destinan las instituciones educativas de nivel superior en favor de contribuir con el desarrollo y solución de diferentes problemas dentro de una sociedad, por medio de la generación de políticas y 
normas institucionales. Frente a esto, la presente investigación tuvo como objetivo, el evaluar la responsabilidad social universitaria de la Universidad Nacional de San Martin sede Tarapoto en el año 2016, para ello fue necesario el contar con teorías que respalden las acciones a realizar, por lo que luego de haber recopilado una serie de teorías, se llegó a establecer como autor principal a Vallaeys y Sasia, (2014), de quien se establecieron cuatro dimensiones, a partir de las cuales se lograron desarrollar un cuestionario con una serie de preguntas, a ser aplicadas a los colaboradores especificados en la muestra. De esta manera, tras aplicar los cuestionarios, tabularlos y posteriormente presentar los resultados en tablas y figuras, se llegó a determinar que tanto la variable, como sus respectivas dimensiones son calificadas como adecuadas, ya que la institución se muestra preocupada por el desarrollo de proyectos y actividades en beneficio de la sociedad, haciendo participes a todos sus integrantes. Estos resultados al ser comparados con el trabajo de (Gaete, 2013), presenta diferencias importantes, como el hecho de que en su investigación no estudió aspectos concretos de la responsabilidad social universitaria, sino que por el contrario el estudio de forma general, llegando a determinar, que esta es vista como inadecuada debido a que los egresados de la universidad no presentan mayor empleabilidad.

La gestión institucional de una institución, constituye el conjunto de actividades realizadas de manera interna basándose en el principio de la planificación, y cuyas repercusiones buscan el bienestar de la organización, así como de sus demás elementos. Es así que, en la presente investigación, otro de los objetivos fue el de evaluar el estado en el que se encuentra la gestión institucional de la Universidad Nacional de San Martin sede Tarapoto, para lo cual se necesitó de teorías concernientes al tema en cuestión, llegando a tomar la teoría de Elera, (2010), quien cita a Huari, (2007), para determinar una serie de dimensiones e indicadores gracias a los cuales fue posible la elaboración de un cuestionario. Por medio de la aplicación y posterior tabulación de los cuestionarios, se llegó a establecer que la gestión institucional de la universidad es calificada como adecuada, discrepando de esta manera con los resultados obtenidos Elera, (2010), quien encuentra a esta dimensión como inadecuada, pues presenta varias deficiencias, entre ellas el hecho de no efectuar una adecuada planeación.

Otro de los objetivos establecidos en la investigación es la de establecer una relación entre la dimensión impacto organizacional con la gestión institucional de la Universidad 
Nacional de San Martin sede Tarapoto, para ello, toda la información recopilada a través de los instrumentos creados con las teorías de Vallaeys y Sasia, (2014), y Elera, (2010), fueron sometidas a comparación por medio del programa SPSS-21, llegándose a determinar que pese a existir una relación entre los elementos comparados, no es muy significativa la incidencia que tiene la dimensión sobre la variable.

Otro objetivo de la investigación es el de determinar o establecer la relación existente entre el impacto educativo con la gestión institucional de la Universidad Nacional de San Martin sede Tarapoto, teniendo como base a autores como (Vallaeys y Sasia, 2014) y (Elera, 2010), de quienes, se estructuraron y tabularon las respuestas a los cuestionarios, por medio del programa estadístico SPSS, el mismo que bajo la prueba de correlación de Rho Spearman llego a determinar la existencia de una relación entre los elementos.

El penúltimo objetivo de la investigación, al igual que las anteriores, fue el de establecer o determinar la relación que existe entre el impacto cognitivo con la gestión institucional de la Universidad Nacional de San Martin sede Tarapoto, para lo cual las teorías empleadas, y de las cuales se desprendieron los cuestionarios fueron de Vallaeys y Sasia, (2014) y Elera, (2010). Los resultados obtenidos de estos instrumentos, concernientes a la dimensión impacto cognitivo y gestión institucional, fueron procesados mediante la prueba de Rho Spearman, llegando a determinar la existencia de una relación significativa entre las variables.

Por último, en la presente investigación se buscó determinar la relación que existe entre el impacto social con la gestión institucional de la Universidad Nacional de San Martin sede Tarapoto, para lo cual, con ayuda del SPSS, y de las teorías propuestas por autores como Vallaeys y Sasia, (2014) y Elera,8 2010), en investigaciones anteriores que sirvieron como antecedentes, se procedió con la aplicación de la prueba de Rho Spearman, el mismo que arrojo una relación significativa entre las variables, llegando así a afirmar que todas las dimensiones, ya sea en mayor o menor medida, presentan una relación con la variable gestión institucional.

\section{CONCLUSIÓN O CONSIDERACIONES FINALES}

De forma general, se llegó a determinar que existe una relación significativa entre las variables, pues el valor sig. Obtenido es de 0.000, así mismo, se evidencia una relación positiva muy fuerte, pues el coeficiente de correlación es de 0.807 ; este coeficiente, al 
ser elevado al cuadrado, evidencia que la variable I incide o afecta en un $65.12 \%$ sobre la variable II.

La responsabilidad social universitaria de la Universidad Nacional de San Martin se encuentra en un estado adecuado con un 59.1\%, debido a que contribuye con la identificación de problemas locales y su posterior solución a través de proyectos que buscan la mejora y el desarrollo de la sociedad.

La gestión institucional de la Universidad Nacional de San Martín, también presenta resultados adecuados con un $56.5 \%$, ya que conlleva la participación adecuada de todos los integrantes, desde docentes hasta estudiantes y personal administrativo.

Los factores relacionados con el impacto organizacional, se relacionan de manera significativa y positiva con la gestión institucional, lo que demuestra que el esfuerzo que se realiza sobre la dimensión afecta a la variable.

En cuanto al impacto educativo se refiere, se determinó que presenta una relación significativa con la gestión institucional, demostrando así la importancia de desarrollar una serie de actividades sobre la dimensión que finalmente afectaran en similar proporción sobre la variable.

El impacto cognitivo, ha demostrado tener una relación significativa con la gestión institucional de la universidad Nacional de San Martin, pues el aprendizaje y las enseñanzas que se imparten a los estudiantes, contribuyen a que estos sean mejores ciudadanos, siendo empleables en la sociedad.

Por último, el impacto social presenta una relación altamente significativa con la Gestión institucional de la Universidade Nacional de San Martin, pues se contribuye en el hecho de que toda la ciudadanía participe de los proyectos, y se dan a conocerlos.

\section{LISTA DE REFERENCIAS}

Aldeanueva, I. et al. (2015). Experiencia iberoamericana en responsabilidad social universitaria. Medellín. [Recurso virtual]. Recuperado de: http://www.funlam.edu.co/uploads/fondoeditorial/ebook/2015/books_gratisExperiencias-iberoamericanas-en-responsabilidad.pdf

Cabral, M. (2012). El proceso de comunicación en el desarrollo de la responsabilidad social empresaria como estrategia corporativa. (Tesis de titulación). Universidad Nacional del Rosario. Santa fe, Argentina. Recuperado de: 
http://www.fcpolit.unr.edu.ar/wp-content/uploads/Tesina-Ma.-In\%C3\%A9s-

Cabral.pdf

Castillo, F. (2007). Análisis de la responsabilidad social ejercida por el centro de entrenamiento pesquero de Paita: Propuesta de desarrollo. (Tesis para magister). Pontificia Universidad Católica del Perú. Lima, Perú. Recuperado de: http://tesis.pucp.edu.pe/repositorio/bitstream/handle/123456789/117/castillo_pal acios_freddy_analisis_responsabilidad_social.pdf?sequence $=1$

Castro, A. (2014). Modelo de Responsabilidad Social UNMSM: Documento de Trabajo. (Versión II). Lima: Universidad Nacional Mayor de San Marcos. [Recurso virtual]. Recuperado de: http://viceacademico.unmsm.edu.pe/wpcontent/uploads/2014/10/Modelo-responsabilidad-social.pdf

Cea, R. (2010). La responsabilidad social corporativa en las entidades bancarias de la Unión Europea. Análisis empírico y propuesta de modelo normalizado. (Tesis para doctorado). Universidad Autónoma de Madrid. Madrid, España. Recuperado de: https://repositorio.uam.es/bitstream/handle/10486/4183/28409_cea_moure_rami ro.pdf? sequence $=1$

Domínguez, C. y Ramos, J. (2012). La Responsabilidad Social Universitaria en la Educación a Distancia. Chimbote, Perú: Editorial Universidad Católica Los Ángeles de Chimbote. Recuperado de: http://virtualeduca.org/documentos/observatorio/oevalc_2012_(rsu).pdf

Domínguez, M. (2009). "Responsabilidad social universitaria". Humanismo y trabajo social. 8 (2009): 37-67. Redalyc. Web. Enero 31 del 2011.

Elera, R. (2010). Gestión institucional y su relación con la calidad del servicio en una institución educativa pública de callao. (Tesis de titulación). Universidad San Ignacio de Loyola. Lima, Perú.

Elías, P. y Vila, E. (2014). “Análisis de la responsabilidad social universitaria de la Pontifica universidad Católica del Perú en el proyecto construcción de viviendas seguras y saludables, para las familias afectadas por el sismo en el centro poblado La Garita - Chincha”. Pontifica Universidad Católica del Perú. Lima. Perú. 
Gaete, R. (2013). Responsabilidad social universitaria: Una nueva mirada a la relación de la universidad con la sociedad desde la perspectiva de las partes interesadas. Partes interesadas. Un estudio de caso. (Tesis para doctorado). Universidad de Valladolid. Valladolid, España. Recuperado de: https://uvadoc.uva.es/bitstream/10324/923/1/TESIS148-120417.pdf

Gil, E. (2013), en su tesis "La responsabilidad social universitaria desde la perspectiva ambiental: universidad y desarrollo sustentable". Universidad Nacional Autónoma de México. México.

Instituto Dominicano de Evaluación e Investigación de la Calidad Educativa-IDEICE (2011). Proceso de gestión institucional y pedagógica. [Recurso virtual]. Recuperado de: http://www.ideice.gob.do/index.php/programas/procesos-degestion-institucional-y-pedagogica

Martí N., Juan J. et al. (2011). "Responsabilidad social universitaria: acción aplicada de valoración del bienestar psicológico en personas adultas mayores institucionalizadas". Polis: revista académica de la Universidad Bolivariana. 18 (2008): 1-13. DIalnet. Web. Enero 31 del 2011.

Martínez, P. (2009). La responsabilidad social empresarial como base de la estrategia competitiva de HZX. (Tesis para titulación). Pontificia Universidad Javeriana. Bogotá, Colombia. Recuperado de: http://www.javeriana.edu.co/biblos/tesis/economia/tesis242.pdf

Molocho, N. (2010). Influencia del clima organizacional en la gestión institucional de la sede administrativa UGEL $N^{\circ}$ 01- Lima Sur-2009. (Tesis para magister). Universidad Mayor de San Marco. Lima, Perú. Recuperado de: http://cybertesis.unmsm.edu.pe/bitstream/cybertesis/3230/1/Molocho_bn.pdf

Olarte, V. (2012). Estudio del grado de desarrollo de la responsabilidad social corporativa a través de las memorias de sostenibilidad y de las audiencias en televisión. (Tesis para doctorado). Universidad de la Rioja. Logroño, España.

Ruiz, M. (2009). La gestión institucional del IEAEY y su incidencia en la educación de jóvenes y adultos maya hablantes en contextos bilingües: maya-castellano. (Tesis para magister). Universidad Nacional de San Simón. Cochabamba, Bolivia. 
Ruiz, E. (2015). Gestión institucional: conceptos introductorios. [Recurso virtual]. Recuperado de: http://www.educ.ar/sitios/educar/recursos/ver?id=91901

Siglo 21 (2013). Responsabilidad Social Universitaria (RSU). Universidad Siglo 21. [Recurso virtual]. Recuperado de: http://www.21.edu.ar/responsabilidadsocial.html

UNESCO-Perú (2011). Manual de Gestión para Directores de Instituciones Educativas. ( $1^{\text {a }}$ ed.). Perú: Depósito Legal en la Biblioteca Nacional del Perú No 2011-04369.

Vallaeys, F. De la Cruz C. y Sasia, P. (2014). Manual de primeros pasos en responsabilización social universitaria: Construyendo ciudadanía en universidades responsables.

Vargas, D. (2012). Gestión pedagógica del trabajo docente a través de grupos cooperativos. Pontificia universidad católica del Perú. Lima 